\section{OHP-009 ANALYSIS OF SUGAMMADEX EXPENDITURE AFTER ITS INTRODUCTION INTO CLINICAL PRACTISE IN A FRENCH UNIVERSITY HOSPITAL}

doi:10.1136/ejhpharm-2013-000276.383

'S Khiter, "I May, ${ }^{2} \mathrm{C}$ Meistelman, ${ }^{2 T}$ Fuchs-Buder. ${ }^{1}$ Chu Nancy Hôpitaux de Brabois, Pharmacy, Vandoeuvre les Nancy, France; ${ }^{2}$ Chu Nancy Hôpitaux de Brabois, Anesthesia, Vandoeuvre les Nancy, France

Background The launch on the market of a new drug is always an important event for a specialty, particularly when the mechanism of action is completely new. It is the case with sugammadex, a cyclodextrin, the first selective relaxant binding agent. It binds and holds within its lipophilic core only the non-depolarizing steroidal muscle relaxants rocuronium and vecuronium. This novel agent acts ten times more rapidly than neostigmine without the need to administer atropine concomitantly.

Purpose To determinate how the arrival of sugammadex has changed the management of neuromuscular blockade in everyday practise and to evaluate the additional cost caused by the use of this drug in all the hospital departments and especially in the department of anaesthesia.

Materials and Methods We conducted a retrospective study over two years' use of sugammadex from January 2010 to December 2011. All the consumption data were extracted from the PHARMA software.

Results During the period of the study, the use of rocuronium increased by $110 \%$, with an additional cost of about $47 \%$, explained by the increase in surgery over 2011 (3\%), and the increased use of sugammadex $(+127 \%)$.

An additional cost $(€ 70,092.84)$ due to the change in practise (neuromuscular block + recovery) was observed. It represents an average increase of $37.4 \%$ over all hospital departments.

In the department of anaesthesia, the use of rocuronium increased by $31 \%(+€ 2,055)$, but did not generate an increase in cost, because the use of other neuromuscular blocking agents (benzylisoquinolines and suxamethonium) decreased between 2010 and 2011.

The number of vials of neostigmine requested from the pharmacy decreased by $37 \%$, while the number of vials of sugammadex increased by $102 \%$.

The additional cost in this department was estimated at $25 \%$; expenditure increased from $€ 68,291.57$ in 2010 to $€ 85,334.63$ in 2011 , caused specifically by the change in neuromuscular block recovery practises.

These results agree with those of Raft et al, 2010, who proved that the increased expenditure was mainly due to the new neuromuscular block recovery practises (€658 to $€ 28,225$ between 2009 and 2010).

Conclusions The introduction of sugammadex into clinical practise joins a quality assurance programme, something new to improve patient safety. However, there are currently pharmacoeconomic barriers to the widespread introduction of sugammadex and further clinical trials will inform the debate concerning cost-effectiveness.

No conflict of interest.

\section{OHP-010 ANALYSIS OF THERAPEUTIC PLANS FOR PATIENTS WITH MULTIPLE SCLEROSIS AT SALERNO UNIVERSITY HOSPITAL: FIRST RESULTS}

doi:10.1136/ejhpharm-2013-000276.384

${ }^{1} \mathrm{~N}$ Ciociano, ${ }^{1} \mathrm{~F}$ Romano, ${ }^{2} \mathrm{G}$ Lombardi, ${ }^{2} \mathrm{M}$ Alfieri, ${ }^{2} \mathrm{M}$ Elberti, ${ }^{2} \mathrm{~L}$ Grisi. ${ }^{1}$ Università degli Studi di Salerno, Scuola di Specializzazione in Farmacia Ospedaliera, Fisciano (Sa), Italy; ${ }^{2}$ Azienda Ospedaliera Universitaria di Salerno, Struttura Complessa di Farmacia Salerno, Italy
Background The Pharmacy Division of Salerno University Hospital distributes medicines to patients referred to the Provincial Centre for Multiple Sclerosis, and since November 2011 has begun monitoring treatment plans to probe the degree of pathology more deeply and patients' use of the drugs.

Purpose The study draws a general profile of patients in the first six months of monitoring.

Materials and Methods Monitoring the treatment plans presented in period 15/11/2011-15/05/2012, the total number, age and sex prevalence of patients were extrapolated, which were classified into: new diagnosis or following a therapeutic programme; severity of neurological disability, according to the Expanded Disability Status Scale (EDSS); drugs used; therapeutic switches; recent interruptions; association with neurological drugs.

Results 165 patients were being assisted, mean age $44 \pm 10$ years. 115 were females. $5 \%$ of the subjects correspond to new diagnoses; $67 \%$ were following a therapeutic programme. $77.94 \%$ had an EDSS score in the range $0.0-3.0 .5 .4 \%$ had scores over 7.0. Patients were starting or continuing treatment with the following medicines: interferon B1a $30 \mathrm{mcg} / 0.5 \mathrm{ml}$ solution for injection (34\%); interferon B1b $250 \mathrm{mcg} / \mathrm{ml}$ solution for injection (24.2\%); interferon B1a $44 \mathrm{mcg} / 0.5 \mathrm{ml}$ solution for injection (13.3\%); interferon B1a $22 \mathrm{mcg} / 0.5 \mathrm{ml}$ solution for injection (2.5\%); glatiramer $20 \mathrm{mg} / \mathrm{ml}$ solution for injection (23\%); fingolimod $0.5 \mathrm{mg}$ capsules $(3 \%)$. Of the subjects in continuation, $30 \%$ were taking interferon $\mathrm{B} 1 \mathrm{a}, 16.4 \%$ glatiramer. $28 \%$ changed treatment because of new neurological abnormalities (50\%), recurrent relapses $(37 \%)$, problems of adherence to the previous regimen $(12 \%)$. One patient each discontinued interferon B1b $250 \mathrm{mcg} / \mathrm{ml}$ and glatiramer due to elevated transaminases. More patients were switched from glatiramer to interferon B1b (33.3\%). 20\% were also taking neurological drugs such as escitalopram $10 \mathrm{mg}(20 \%)$, baclofen $25 \mathrm{mg}(16 \%)$, carbamazepine $400 \mathrm{mg}(10 \%)$

Conclusions A high percentage of patients emerge who, despite having neurological deficits, are living independently. In this stage there may be less full awareness of the disease, and pharmacists, with personalised counselling, can detect, correct and prevent poor compliance

No conflict of interest.

\section{OHP-011 APPLICATION OF BENCHMARKING TECHNIOUES TO HOSPITAL PHARMACY PRACTISE}

doi:10.1136/ejhpharm-2013-000276.385

A Lustig, S Kerekesh, 0 Rubanov, R Sokol, S Aflalu. Barzilai Medical Center, Pharmacy, Ashkelon, Israel

Background Benchmarking is a process designed to discover best practise through a comparison of various competing methods.

The use of drug benchmarking can identify problems in health team practise, yield a clearer understanding of competitor hospitals and aid in establishing attainable goals.

Purpose To identify differences in drug expenditure between two hospitals.

Materials and Methods Two hospitals with the same number of occupied beds, size and medicines procurement systems were evaluated for drug expenditure. Analysis included financial measurements: expenditure per hospitalisation day, per patient, cost grading (Pareto), drug inflation index and cost analysis by a time \& motion study. Clinical measurements used policies of checking the suitability of drug use and antimicrobial streamlining programmes.

Results Hospital A' drug expenses (+11.5\%) and cost/patient $(+35 \%)$ were higher than in hospital B', the main differences being attributed to the use of infusions and antibiotics. A comparison between IV infusions showed a higher expenditure in hospital $A^{\prime}$ (48\%) compared to B'; differences were attributed to the practise 
models of drug preparation and administration. In hospital A' all drugs were diluted in a minibag under aseptic conditions, while in hospital B', nurses diluted the drug on the wards and administered it through a buretrol device. A cost analysis time \& motion study was performed to find out the cost of each practise model; 11.3 Euro/ unit in hospital A'versus 13 Euro in hospital B'. Hospital A' used PTZ' treatment based on a streaming system \& antibiogram assistance, while in B', use was according to physician approval and a system of switching.

Conclusions This survey has several limitations: the difficulty of accurately reflecting prescribing practises, equipment and patient case mix. Nevertheless, the benchmarking exercise provided valuable data, which can be used to target key areas for cost control and performance.

No conflict of interest.

\section{OHP-012 ASSESSMENT OF UNIFIED INHALATION GUIDANCE DOCUMENTS FOR DIFFERENT INHALERS AND THE INFLUENCE OF AGE ON INHALATION TECHNIOUE}

\section{doi:10.1136/ejhpharm-2013-000276.386}

${ }^{1} \mathrm{R}$ Ono, ${ }^{1} \mathrm{~A}$ Hosomi, ${ }^{2} \mathrm{~T}$ Horie, ${ }^{3} \mathrm{~J}$ Onozato, ${ }^{1} \mathrm{~T}$ Hashita, ${ }^{4} \mathrm{~T}$ Araki, ${ }^{1} \mathrm{~K}$ lizuka, ${ }^{4} \mathrm{~T}$ Nakamura, ${ }^{4} \mathrm{~K}$ Yamamoto, ${ }^{5} \mathrm{~K}$ Dobashi. 'Gunma University Hospital, Pharmacy, Maebashi-shi, Japan; ${ }^{2}$ Maebashi Red Cross Hospital, Respiratory Medicine, Maebashi-shi, Japan; ${ }^{3}$ Maebashi Red Cross Hospital, Pharmacy, Maebashi-shi, Japan; ${ }^{4}$ Gunma University Graduate School of Medicine, Clinical Pharmacology, Maebashi-shi, Japan; ${ }^{5}$ Gunma University Graduate School of Health Sciences, Maebashi-shi, Japan

Background When using an inhaler for asthma or chronic obstructive pulmonary disease, the correct inhalation technique is essential for obtaining the desired effect. However, the development of different types of inhalers has led to inhalation techniques that differ greatly among devices. Therefore, we prepared the 'Unified Inhalation Guidance Documents' (UIGDs) in cooperation with hospitals and pharmacies in our region for each available type of inhaler.

Purpose To assess the benefits and problems of the UIGD.

Materials and Methods A total of 165 Japanese patients who received inhalation therapy from June 2011 to September 2012 were enrolled, and 213 points regarding the inhalation technique with 8 types of inhalers were obtained. The inhalation technique of patients who received guidance based on the UIGD for the use of inhalers was assessed by scoring inhalation skill. In addition, we assessed the impact of age on the acquisition of inhalation technique.

Results We found that 86 cases (40.4\%) showed problems with inhalation technique. In particular, patients using a Breezhaler (4/5) and Turbuhaler (18/37) had a high rate of problems with their technique. Problems were more frequent in patients aged 65 or over (older) $(63 / 146,43.2 \%)$ compared with other patients (younger) $(23 / 67,34.3 \%)$. In particular, for patients using a Turbuhaler, more older patients $(10 / 14,71.4 \%)$ than younger patients $(8 / 23,34.8 \%)$ had trouble with technique.

Conclusions Around $40 \%$ of patients who received guidance based on the UIGD for the use of inhaler devices had trouble with their inhalation technique. Therefore, the UIGDs for the Breezhaler and Turbuhaler should be reassessed. Because the use of some types of inhalers was difficult for older patients, developing an inhaler and guidance specifically for older patients should be considered.

No conflict of interest.

\section{OHP-013 BLEEDING IN CARDIAC SURGERY: USE OF BLOOD COAGULATION FACTORS}

doi:10.1136/ejhpharm-2013-000276.387

V Calmels, A Recurt-Carrere, E Divol, B Sallerin. Chu Toulouse - Rangueil, Pharmacy, Toulouse, France
Background There are few references or publications about the management of bleeding during cardiac surgery. Practices are influenced by the availability of drugs and equipment and are team-dependant.

Off-label use of blood coagulation factors (BCFs) has become a major public health and financial concern.

Purpose To explain the increasing use of BCFs, during cardiac surgery under Extracorporeal Circulation (ECC), in a University Hospital setting.

Materials and Methods We assessed the amounts of BCFs and Transfused Blood Products (TBPs) used between 2009 and 2011 and compared these figures to the number of operations using ECC.

Results During this 2 years, the workload, in number of operations requiring ECC, increased by only $3 \%$ (with a decrease of $11 \%$ in emergencies).

The use of TBP increased $3 \%$.

In the same time, BCF prescriptions increased dramatically (representing a cost of Euro 270,000). The analysis of quantities dispensed shows:

- An increase of over 138\% for Prothrombin Complex Concentrates (PCCs)

- Increase of over $586 \%$ for fibrinogen (Fg)

- Increase of over $102 \%$ for activated factor VII

National figures for the same period were:

- $23 \%$ increase for PCC

- $70 \%$ increase for $\mathrm{Fg}$

- $4 \%$ increase for FVII

Conclusions The increased use of these factors can be explained by changes in local professional practise. In order to standardise and justify the use of these costly products, multidisciplinary meetings (anaesthetists, biologists, cardiac surgeons and pharmacists) are taking place. A procedure for the management of bleeding during cardiac surgery is in development. This document should contribute to the improvement of care in terms of therapeutic efficiency and safety.

No conflict of interest.

\section{OHP-014 CLINICAL AND FINANCIAL EFFECTS OF THE USE OF A THERAPEUTIC EOUIVALENCE PROGRAMME IN A TERTIARY HOSPITAL}

doi:10.1136/ejhpharm-2013-000276.388

'C García, ${ }^{2} \mathrm{C}$ Alarcón, 'I Cañamares, 'I Escribano, 'MJ Jiménez, 'JM Ferrari. 'Hospital Universitario 12 de Octubre, Pharmacy, Madrid, Spain; ${ }^{2}$ Hospital Universitario Virgen de las Nieves, Pharmacy, Granada, Spain

Background A Therapeutic Equivalence Program (TEP) assembles clinically equivalent drugs and defines the best therapeutic alternative included in the hospital's pharmacotherapeutic guide to drugs not included (DNI).

Purpose To measure the clinical and financial impact of a TEP in a tertiary hospital.

Materials and Methods Descriptive observational study conducted between November 2011 and January 2012. During the transcription and validation of the prescriptions of clinical units that work with unit-dose drug distribution system, pharmacist applied the TEP and notified the physician of the substitution in writing. The prescription of an unincluded was only retained if there was a clinical justification that made substitution impossible.

The variables collected were: rate of substitution proposals accepted and rejected, justifications for not performing the substitution, cost of the DNI, cost of the therapeutic equivalent per hospital stay and percentage of therapeutic equivalents prescribed at discharge. 